\title{
Modelo de atención para el tratamiento de pacientes con osteoartritis del primer al tercer nivel
}

\author{
Model of care for the treatment of patients with osteoarthritis from the first to the third level \\ Rodríguez-Skewes E, ${ }^{*}$ Quiñones-Díaz Terán MÁ,* Negrete-Corona J,* \\ Moralez-Xolalpa G, ${ }^{*}$ Negrete-Camacho JJ, ${ }^{*}$ Bello-Cárdenas DE, ${ }^{*}$ Solano-Gutiérrez N, ${ }^{*}$ \\ Camacho-Ruíz Y, ${ }^{*}$ Rodríguez-Arellano ME, Martínez-Rodríguez NL, ${ }^{\S}$ Cruz-Sánchez DX*
}

Servicio de Ortopedia, Hospital Regional «Lic. Adolfo López Mateos»,

Instituto de Seguridad y Servicios Sociales de los Trabajadores del Estado.

RESUMEN. Introducción: La osteoartritis en México es una de las 10 causas más frecuentes de discapacidad. El diagnóstico temprano y la detección de factores de riesgo son determinantes para el tratamiento. La organización institucional establece las pautas terapéuticas de acuerdo con cada nivel de atención, pero no se logra un manejo efectivo. Material y métodos: Se realizó un estudio prospectivo, piloto, de intervención, clínico conformado por pacientes diagnosticados con diferentes grados de osteoartritis de rodilla en el primer, segundo y tercer nivel de atención, con un modelo integrador que incluyó un grupo de profesionales para la intervención de la evaluación nutricional, fisioterapéutica, social y sicológica desde el primer nivel. Resultados: La intervención de un grupo de atención multidisciplinaria permite una correcta evaluación y asignación del nivel de atención optimizando los recursos humanos y materiales. La participación de diferentes disciplinas en nutrición, sicología, trabajo social, fisioterapia y rehabilitación modifica el estilo de vida global al involucrar a los propios pacientes en su tratamiento. El grupo de intervención tuvo mejoras en la escala de dolor análogo visual, arcos de movilidad, bajo índice de masa corporal y mejoría en el aspecto sicológico. Conclusiones: El modelo de atención propuesto demuestra
ABSTRACT. Introduction: Osteoarthritis in Mexico is one of the ten most frequent causes of disability. Early diagnosis and detection of risk factors are determinant for treatment. The institutional organization establishes therapeutic guidelines according to each level of care, but effective management is not achieved. Material and methods: A prospective, pilot, interventional, clinical study was conducted, which included patients diagnosed with different degrees of knee osteoarthritis in the first, second and third level of care, with an integrative model that includes a group of professionals for the intervention of nutritional, physiotherapeutic, social and psychological evaluation from the first level. Results: The intervention of a multidisciplinary care group allows a correct evaluation and assignment of the level of care, optimizing human and material resources. The participation of different disciplines in nutrition, psychology, social work, physiotherapy and rehabilitation modifies the global lifestyle by involving the patients themselves in their treatment. The intervention group had improvements in the visual analog pain scale, mobility arcs, low body mass index and improvement in the psychological aspect. Conclusions: The proposed model of care demonstrates that implementation in the institution and

\section{Nivel de evidencia: IV}

* Servicio de Ortopedia, Hospital Regional «Lic. Adolfo López Mateos», ISSSTE, Ciudad de México, México.

` Laboratorio de Medicina Genómica, Hospital Regional «Lic. Adolfo López Mateos», ISSSTE, Ciudad de México, México.

${ }^{\S}$ Departamento de Investigación en Salud Comunitaria. Hospital Infantil de México «Federico Gómez». Ciudad de México, México.

Correspondencia:

Diana Xóchitl Cruz-Sánchez

Cerrada Durango Núm. 7, Col. Progreso, Alcaldía Álvaro Obregón, C.P. 01080.

E-mail: dtdianac@gmail.com; d.cruz@issste.gob.mx

Recibido: 13-11-2019. Aceptado: 14-09-2021.

Citar como: Rodríguez-Skewes E, Quiñones-Díaz TMÁ, Negrete-Corona J, Moralez-Xolalpa G, Negrete-Camacho JJ, Bello-Cárdenas DE, et al. Modelo de atención para el tratamiento de pacientes con osteoartritis del primer al tercer nivel. Acta Ortop Mex. 2021; 35(4): 331-340. https://dx.doi.org/10.35366/103313 
que la implementación en la institución y en cada unidad clínica de atención debe considerarse para mejorar los resultados.

Palabras clave: Osteoartritis, tratamiento, modelo, integración, resultados.

\section{Introducción}

El seguro de salud institucional tiene por objeto proteger, promover y restaurar la salud de los derechohabientes, para lograr esta encomienda implementa los programas de organización buscando distribuir de manera uniforme los recursos; sin embargo, por diversas razones y circunstancias, la atención hospitalaria y de especialidad rebasan la atención en el primer nivel, condicionando una pirámide de infraestructura en salud invertida, donde se tiende a resolver incluso los problemas de salud más sencillos en el ámbito hospitalario y con recursos humanos especializados; esto con los consecuentes incrementos en los costos de la atención. De este modo, los esfuerzos que el Instituto de Seguridad y Servicios Sociales de los Trabajadores del Estado (ISSSTE) ha realizado por reorganizar y ordenar la atención médica siguen teniendo malos resultados. Sin duda es imperativo llevar a cabo acciones en pro de la distribución adecuada. Actualmente, es de capital importancia reconocer que el proceso de envejecimiento por el que atraviesa nuestro país en general no exime a la población institucional, en particular el porcentaje de derechohabientes mayores de 65 años, pues es dos veces superior al porcentaje correspondiente a nivel nacional. Esto ejerce una enorme presión sobre los servicios de salud, ya que los adultos mayores generan mayor demanda de atención que el resto de la población y sus tratamientos son complejos y costosos. ${ }^{i}$ Asimismo, las patologías degenerativas del sistema musculoesquelético ocupan un lugar preponderante, específicamente la osteoartritis, que es la forma más común de artritis que afecta a hombres y mujeres en la plenitud de la edad. Los estudios de prevalencia reportan la afectación de rodilla en $10 \%$ en el sexo masculino y en $13 \%$ en el femenino. Su base fisiológica es la pérdida progresiva del cartílago articular. Las principales manifestaciones clínicas son el dolor y la limitación funcional. ${ }^{1,2,3}$

En la última década, el manejo terapéutico de la osteoartritis se enfoca principalmente en el tratamiento farmacológico, ${ }^{4,5,6}$ donde la gama de tratamientos es diversa y permite disminuir sus efectos e incluso modificar su curso, pero los resultados son desfavorables en el costo-beneficio, debido a esto se considera una terapia insuficiente. En la búsqueda por optimizar los recursos con los que cuenta el ISSSTE, nuestra propuesta es la integración de un modelo de atención multidisciplinario para la atención de los pacientes con

\footnotetext{
${ }^{i}$ http://sgm.issste.gob.mx/medica/plan_rector/Plan_Rector_Fin.pdf
}

in each clinical care unit should be considered to improve outcomes.

Keywords: Osteoarthritis, treatment, model, integration, results.

osteoartritis del primer al tercer nivel de atención, por lo que es necesario un abordaje terapéutico multidimensional en el cual se llevará a cabo la integración del equipo en el que participan cuatro disciplinas, muy vinculadas con los procesos de vida que atraviesan los seres humanos de manera cotidiana, así como del equipo de profesionales con estudios en trabajo social, nutrición, sicología y rehabilitación sin descartar la parte médica en que están inmersos los sujetos de estudio. ${ }^{7,8}$

La evidencia en la literatura reconoce que este tipo de intervenciones, donde se observa una modificación de los factores de riesgo de desarrollar la enfermedad, disminuyen la sintomatología y favorecen la función de las articulaciones afectadas, incluso podría modificar su curso, tal es el caso del fortalecimiento muscular y el control de peso. ${ }^{7,8,9,10}$ En cuanto al control de peso, se ha observado que disminuir en $5 \%$ el peso corporal y mantener un buen programa de alimentación y ejercicios son factores determinantes. $1,9,10,11$

En diferentes estudios se ha observado que para el buen control y seguimiento de los pacientes con osteoartritis la atención sicológica tiene un papel importante en el tratamiento no farmacológico debido a que este padecimiento genera una carga mayor de ansiedad, por lo que este tipo de intervenciones con manejo sicológico trae beneficios a largo plazo $^{12,13}$ y dentro de la vida real, involucrando el entorno social y familiar. ${ }^{7,8,14}$

Actualmente el ISSSTE cuenta con estos profesionales en su plantilla de trabajadores para cada una de las unidades de atención en los diferentes niveles de estratificación. Fomentar la preocupación por este grupo de enfermos con un enfoque integral echando mano de estos recursos necesariamente impactará en la calidad de atención y por ende, en la óptima distribución de los insumos. Cuando se aborda al paciente de manera integral desde el primer nivel de atención, se pueden limitar los envíos indiscriminados tanto al segundo como al tercer nivel de atención y aun cuando el paciente necesite de intervención especializada, el fomento de los cambios en sus hábitos generará mejor preparación en el caso de recibir un tratamiento quirúrgico de cualquier índole, mejorando el pronóstico.

Considerando los antecedentes señalados, se propone una intervención para evaluar la eficacia de un programa multidisciplinario que integre al grupo de trabajo social, nutrición, sicología y rehabilitación que permita mejorar las manifestaciones de dolor y conductuales del paciente. ${ }^{1,2,7,8}$ 


\section{Material y métodos}

Se realizó un estudio prospectivo, piloto, de intervención, clínico, de cohorte, aplicado en el que se incluyeron pacientes diagnosticados con diferentes grados de osteoartritis de la rodilla.

Cálculo de la muestra. La información que se tomó para calcular la muestra fue la prevalencia de osteoartritis en adultos mayores $(\sim 50 \%)$ y el efecto de una intervención de terapia física en la mejoría esperada tomada de otros estudios. Se usó una fórmula de diferencia de medias en la que se integraron un valor alfa de 0.05 , un valor beta de 0.2 , un poder de 0.8 y se adicionó $20 \%$ de una diferencia de medias a la cifra estimada para compensar las pérdidas que con frecuencia ocurren en los ensayos clínicos. La muestra se estimó en 60 pacientes por grupo con el fin de detectar el tamaño mínimo del efecto. Los tamaños mínimos del efecto se definieron a partir de los resultados observados en otros estudios. Para este estudio se detectó un poder estadístico de la muestra de estudio con un valor alfa de 0.05 y un valor de correlación de $90 \%$ fue de 0.847 . El estudio se conformó de 60 pacientes que corresponden a la atención de primer nivel institucional, mismo que descansa en la figura de dos clínicas familiares que pertenecen al ISSSTE. En el grupo control se integró a 60 pacientes observados en el tercer nivel de atención que se sometieron al tratamiento de manera tradicional.

Después de la explicación del estudio, a los pacientes que aceptaron participar se les pidió que firmaran una carta de consentimiento informado en presencia de un testigo. Todos los pacientes fueron evaluados por un equipo multidisciplinario. El estudio se conformó por pacientes a quienes se les aplicó el modelo de intervención y por otro grupo donde se observó el manejo tradicional para osteoartritis. El estudio fue aprobado por los comités de investigación, ética y bioseguridad del hospital y se obtuvo el consentimiento informado, de acuerdo con la ley general de salud en materia de investigación en seres humanos (Reglamento de la Ley General de Salud en Materia de Investigación para la Salud).

El equipo de intervención trabajó con los sujetos del primer grupo durante 12 meses y se estableció como tope de consulta general cuatro mediciones.

El procedimiento de la intervención se realizó de la siguiente manera:

1. Entrevista de primera vez para dar información concerniente, firma de consentimiento y primera medición para datos basales.

2. Obtención de mediciones y revisión de resultados.

3. Segunda entrevista y medición.

4. Última intervención con intervalo de dos a tres meses.

En el segundo grupo, como consecuencia de las dificultades encontradas a lo largo del proyecto, se trabajó durante seis meses, acuerdo derivado de las observaciones y recomendaciones del evaluador.
Criterios de selección. Pacientes con diagnóstico de osteoartritis recibidos en los niveles de atención que fueron integrados al grupo de estudio y a los cuales se les realizó la intervención al aplicar el modelo de atención propuesto.

\section{Diseño y desarrollo de la intervención}

El proyecto se dividió en tres etapas. 1. Integración y capacitación del equipo multidisciplinario especializado para el modelo de atención integral de osteoartritis en el ISSSTE, sensibilización y capacitación de las unidades de primer, segundo y tercer nivel de atención. 2. Monitoreo y evaluación de la implementación del modelo de atención integral de osteoartritis en el ISSSTE de las unidades de primer, segundo y tercer nivel de atención. 3. Evaluación del modelo de atención integral de osteoartritis en el ISSSTE.

Se trabajó en dos clínicas institucionales que corresponden al primer y segundo nivel de atención en salud. Ambas seleccionadas por disponibilidad de apoyo. En cada unidad se reclutaron 60 individuos, en la de nivel familiar enviados por médicos generales y familiares, mientras que en la de segundo nivel por especialistas en ortopedia.

Los sujetos integrados en la intervención fueron de ambos sexos con edades a partir de la cuarta década de la vida portadores de dolor articular en cualquiera de los grados de afectación establecidos en la literatura. Todos ellos con afectación de la rodilla. De manera inicial fueron reclutados 79 individuos, de los cuales fueron excluidos ocho por presentar enfermedades con afectación neurológica y relacionadas con la columna vertebral, cinco que no asistieron de manera constante a las entrevistas y mediciones, dos que fueron enviados por su médico tratante a cirugía, tres que recibieron tratamientos invasivos en otras unidades de atención y uno que falleció por complicaciones de enfermedad cardiovascular.

Los profesionales utilizaron herramientas para obtener información mediante entrevista, la aplicación de un cuestionario, el llenado de una hoja de valoración fisioterapéutica, la hoja de escala de Yesavage y una evaluación nutricional del adulto. La intervención en primer nivel tuvo lugar entre Agosto de 2016 y Agosto de 2017. Los sujetos fueron informados de los riesgos para la salud articular asociados con la obesidad y los beneficios de adoptar hábitos saludables de alimentación y actividad física. Dicha información fue otorgada por los especialistas de modelo de atención establecido. El sicólogo hizo una entrevista inicial para establecer un diagnóstico y en cada caso presentó recomendaciones. La trabajadora social realizó un estudio socioeconómico. Los pacientes asistieron a entrevistas y evaluaciones para medición en sesiones de 90 minutos cada dos a tres meses de acuerdo a la disponibilidad de sus horarios, Martes y Jueves en áreas asignadas por autoridades de la clínica en el turno vespertino a partir de las 15:00 horas.

\section{Medición antropométrica}

Una nutricionista integrante del equipo perteneciente al modelo de atención, familiarizado con los procedimientos 
antropométricos internacionales, midió y pesó a los sujetos de estudio tres veces durante el seguimiento con cinta ergonómica para medir circunferencias (SECA 201, England), báscula electrónica de piso de alta capacidad y estabilidad (modelo Seca-813, USA) y estadiómetro portátil (SECA 2131, USA). Las mediciones se realizaron sin zapatos y con ropa ligera, de pie, con los bazos en los costados y cabeza en plano horizontal. Se obtuvo el índice de masa corporal (IMC) y se estableció el peso normal, sobrepeso y obesidad de acuerdo a las normas proporcionadas por la Organización Mundial de la Salud (OMS) con procedimientos internacionalmente aceptados y estandarizados por persona. ${ }^{15}$

\section{Valoración fisioterapéutica}

La selección de los parámetros que permitieran evaluar la eficacia de esta terapia no farmacológica se basó en los objetivos específicos de la intervención y de los resultados reportados en estudios anteriores. ${ }^{15,16,17,18}$ La medición de la amplitud del movimiento articular en cuanto a la determinación de la flexión y extensión articular en grados se realizó utilizando la goniometría siguiendo la biomecánica de la articulación en rodilla. ${ }^{18,19}$ Los resultados que evaluaron la fuerza muscular se obtuvieron a través de la escala de Danniels $(0-5)^{20}$ y para la clasificación del dolor se usó la escala visual análoga (EVA: 0-10). La medición de la movilidad articular se efectuó en grados.

\section{Valoración social}

En primer lugar se determinó el familiograma en los casos que fue posible con la finalidad de conocer el entorno del enfermo. Posteriormente, con la ayuda de las respuestas del cuestionario se tomó en consideración la ubicación de la vivienda y poder adquisitivo, con lo cual se pudo manejar un programa de recomendaciones centrado en el paciente con el mayor aprovechamiento del entorno en todos los niveles. ${ }^{21,22}$

\section{Valoración sicológica}

Los diagnósticos fueron obtenidos de la primera intervención mediante entrevista clínica y cuestionario. ${ }^{23,24,25} \mathrm{Y}$ para la última evaluación se utilizó la escala clinimétrica de Yessavage y la entrevista clínica. Además se incluye una amplia gama de variables para evaluar los resultados secundarios como comorbilidad, institucionalización, uso de servicios de salud, costos de atención de su enfermedad, entre otras, que serán abordadas en otro documento. Datos de seguimiento incluyeron el registro de la mortalidad de los participantes y su causa.

Análisis de los datos. De los datos antropométricos se obtuvieron medias y desviación estándar o sus equivalentes no paramétricos obteniendo medianas e intervalos intercuartílicos.

Para comparar los grupos con el tiempo basal, en cuanto a las variables continuas, se utilizó la prueba ANOVA (variables con distribución normal), prueba de Kruskal-Wallis (para datos sin distribución normal) y la prueba $\chi^{2}$ para los datos categóricos.

El efecto de las variables independientes se analizó comparando el cambio en los parámetros antropométricos y de las diferentes escalas de dolor desde el inicio y hasta el término de la intervención.

Para evaluar el cambio intragrupo en las mediciones se utilizaron modelos de regresión lineal ajustados por cada variable dependiente, edad, sexo y cita; más tarde se obtuvieron las medias ajustadas. Los valores de $\mathrm{p}<0.05$ fueron tomados como estadísticamente significativos para todos los análisis. El análisis se realizó utilizando el paquete estadístico STATA SE v. 12.0 (Stata Corp. College Station. TX. USA).

Se utilizaron estadísticas descriptivas para describir la línea de base de la población de estudio. El peso medio y la altura se ajustaron por edad y sexo mediante regresión lineal múltiple. Para comparar los grupos en la línea de base se utilizó la prueba t para datos dependientes para las variables continuas y la prueba $\chi^{2}$ para los datos categóricos.

Debido a que con frecuencia en este tipo de estudio los participantes no siempre siguen las instrucciones y por lo tanto, la adherencia no es de $100 \%$, consideramos apropiado realizar el análisis de los datos por intención de tratar, en el que se estudian todos los participantes asignados a cada grupo de su adherencia.

Los cambios en las diferentes escalas intragrupo desde el inicio y en el transcurso de las diferentes citas con intervalo de 10 a 12 semanas entre ellas se compararon mediante la prueba t pareada.

Se utilizó un modelo de regresión lineal de efectos mixtos para evaluar el cambio en los diferentes valores dados para el dolor utilizando la escala, la resistencia y fuerza durante el seguimiento. El modelo fue ajustado por las variables fijas de edad y sexo. Se evaluó la interacción entre el grupo de intervención y el tiempo en cada cita. Se calculó la media de cada escala en el grupo de intervención y por tiempo y se realizó un gráfico utilizando análisis marginal. Los valores de $\mathrm{p}<0.05$ se consideraron estadísticamente significativos para todos los análisis (Figura 1).

\section{Resultados}

Para el grupo control se captaron 60 pacientes, para el grupo de intervención iniciaron 79 pacientes, de los cuales 60/79 concluyeron la intervención (Figura 2). La Tabla 1 describe las características de los grupos de estudio. La distribución de hombres y mujeres fue similar en ambos grupos de estudio así como la edad de los pacientes que fue de 60 años.

En el grupo de intervención se usaron mediciones estandarizadas. Se tomaron datos basales de peso, talla, índice de masa corporal y se determinó un diagnóstico para el estado de nutrición en que se encontraban los sujetos: 66.67 con un rango normal, $31.67 \%$ en riesgo de malnutrición y 1.67\% con estado de malnutrición. Esto fue considerado de gran relevancia para el abordaje y para las recomendaciones de la nutrióloga. Como parte de la evaluación médica y la revisión ortopédica 
se observó a la población con diversos grados de desgaste articular u osteoartritis; de la información recabada encontramos que la mayor parte de nuestros pacientes cursaban con datos compatibles por clínica y, en los que fue posible la radiografía, tenían diagnóstico de grado II, después el grado III y por último, en los menos casos, el grado I. La mayoría de nuestros pacientes habían tenido síntomas dolorosos por más de un año e incluso por arriba de ese período y $73.33 \%$ había presentado datos francos de inflamación.

En cuanto a las características antropométricas, los pacientes en la primera cita, que fue el momento basal de la intervención, comenzaron con un peso de $74.24 \mathrm{~kg}$ al inciar la intervención con el grupo de nutrición, en la segunda cita el peso se redujo a $74.07 \mathrm{~kg}$, en la tercera cita de $73.70 \mathrm{~kg}$ y en la cuarta cita de $71.66 \mathrm{~kg}$. Para el índice de masa corporal (IMC) los pacientes comenzaron con un IMC de 30.17 y terminaron con un IMC de 29.39, con un porcentaje de grasa de $35.83 \%$ (Tabla 2).
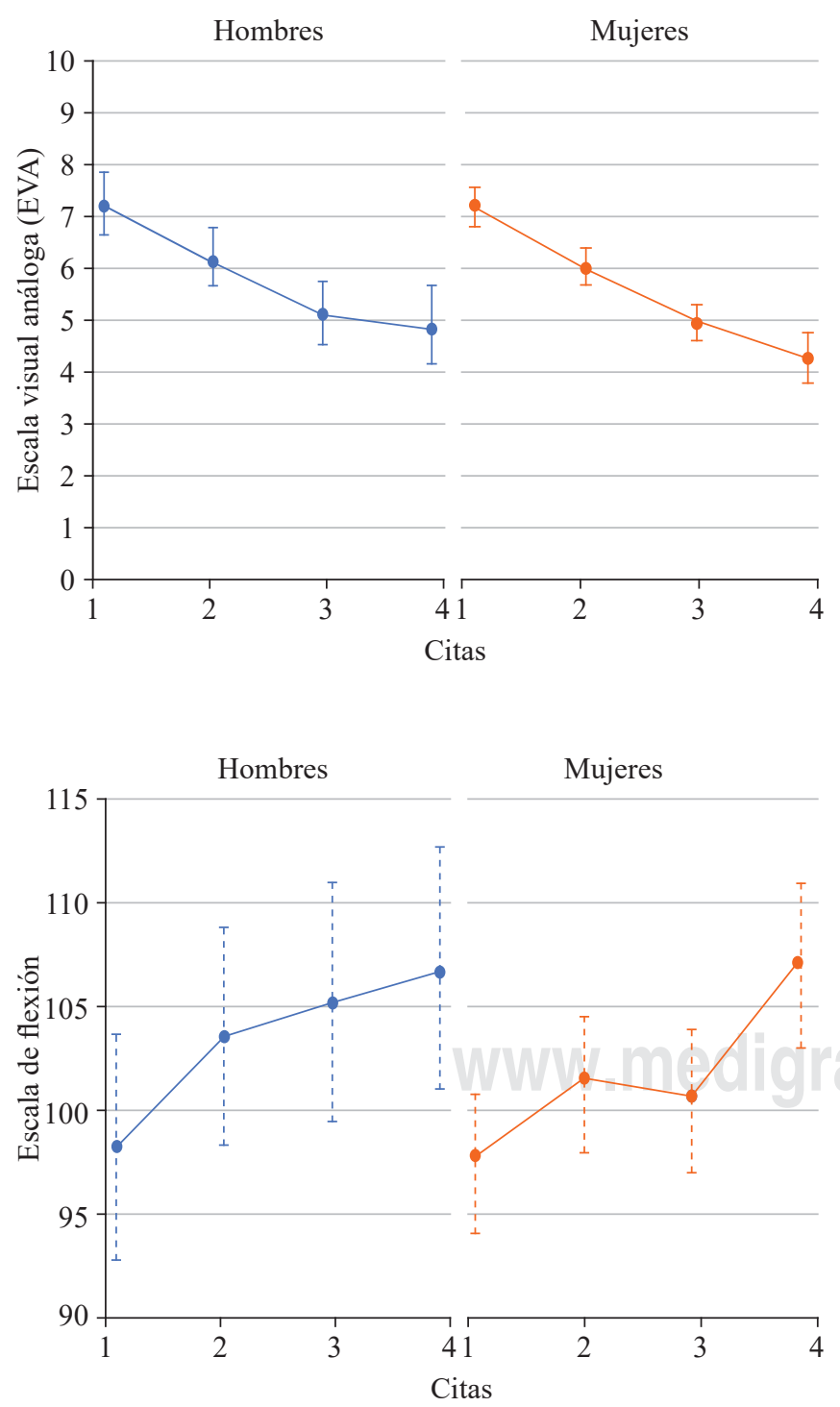

Figura 1: Modelo de efectos mixtos de regresión lineal.
Cambios antropométricos y en las diferentes escalas de valoración mostrados a lo largo de la intervención

Los cambios ocurridos en estos parámetros se muestran en la Tabla 3 en el transcurso de la intervención en cada cita. Durante la intervención los pacientes tuvieron una reducción de peso de $649 \mathrm{~g}$ (no significativo), estimando una pérdida de peso considerable para el efecto clínico que medimos. Con respecto a los cambios en las escalas durante la intervención, se observa una disminución del índice de masa corporal y el porcentaje de grasa, considerada una buena respuesta de la población tomando en cuenta la intervención de la nutrióloga; sin embargo, los cambios no son significativos estadísticamente, pero sí determinantes para el bienestar de los pacientes.

Uno de los puntos más importantes fue precisamente la expresión subjetiva de mejoría al cambiar las condiciones de movilidad de la articulación y con esto una discreta disminución del dolor que clínicamente mejoró la función (Tabla 3).
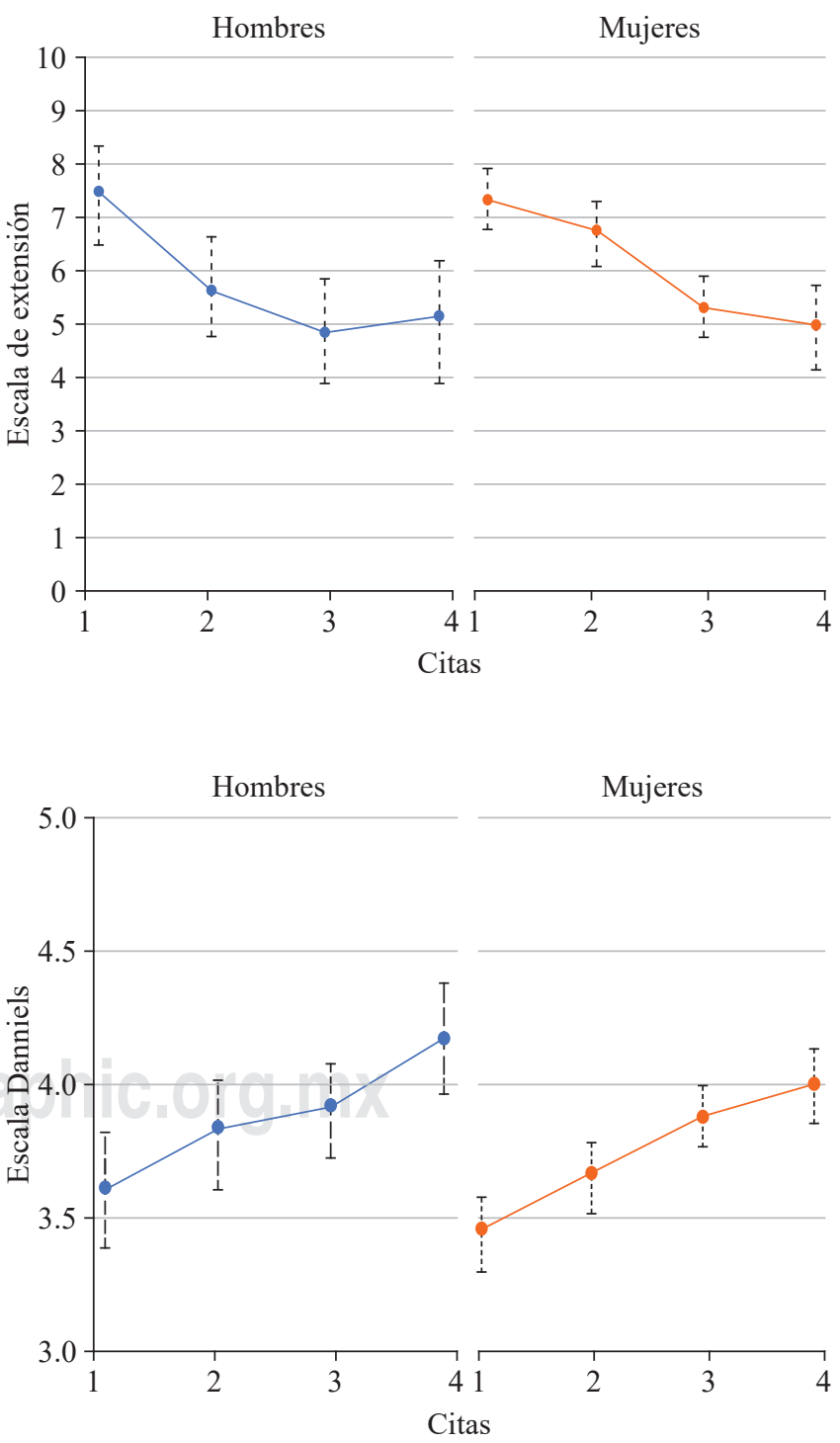


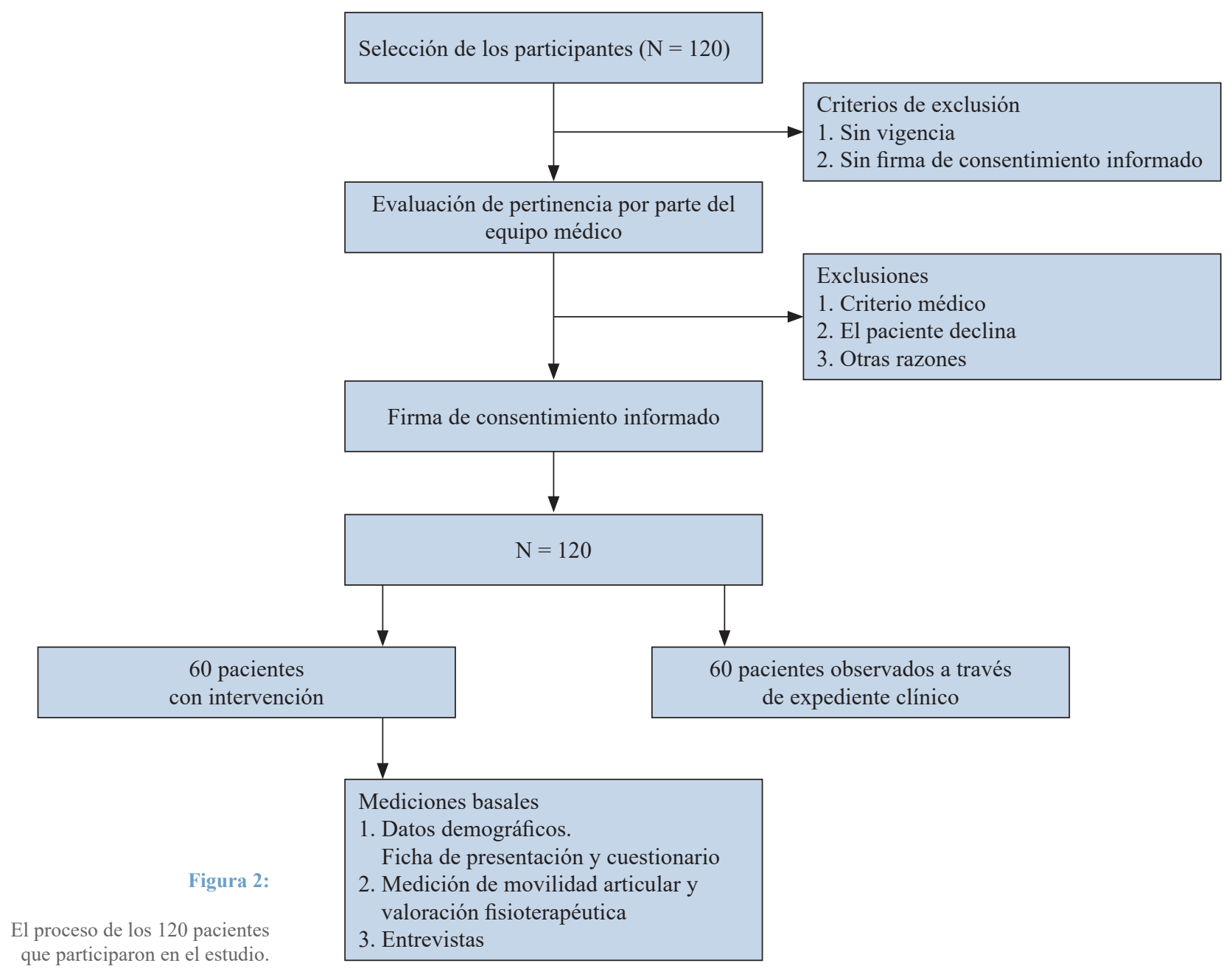

Se muestra un modelo de regresión lineal con efecto mixto, ajustado por edad y sexo y dato basal de cada una de las escalas. Se observa el dato basal y el seguimiento en las diferentes citas durante la intervención. Se muestran las medias ajustadas y el cambio en éstas para cada una de las escalas al inicio y en el transcurso de la intervención. En este modelo se puede observar la disminución en la escala de dolor comenzando con siete de 10 puntos y terminando con cuatro de 10 puntos, es decir, que para la última cita a los 12 meses se presentó una disminución de casi tres puntos. Al final de la intervención observamos aumento en la flexión así como modificación de la extensión (Tabla 4).

\section{Evaluación sicológica}

Durante la primera evaluación clinimétrica de la valoración sicológica, aplicando el cuestionario, se detectaron diagnósticos relacionados a trastornos de sueño, del estado de ánimo y de ansiedad. Se aplicó una intervención consistente en recomendaciones y cambios en el estilo de vida, no se utilizaron medicamentos. Al final del período de estudio, se aplicó la misma evaluación y se registraron los cambios para cada caso.

\section{Discusión}

En cuanto al grupo de observación, identificamos diferentes formas o patrones de atención cuando el paciente no tiene el seguimiento en las clínicas familiares o de especialidades.

Primeramente, son enviados a cualquiera de las opciones del siguiente nivel, de manera indiscriminada incluso a petición del paciente o por recomendaciones externas.

En todos los casos se registra la fecha de atención, los datos personales del enfermo, la edad y el sexo.

En 51\% se tiene registro de talla, 53\% tiene registro de peso, por lo tanto sólo la mitad de esta población es susceptible de cálculo en el IMC.

El número de consultas para atención varía de acuerdo a la selección del tratamiento que queda a cargo del médico tratante. Derivado del tipo de manejo terapéutico diferenciamos el grupo 1 (58.3\%, alto), que recibe de primera intención la viscosuplementación como primera alternativa de tratamiento, del grupo 2 (15\% moderado), que incluye los pacientes sometidos a tratamiento quirúrgico de mínima invasión y del grupo 3 (6.6\% bajo), que se somete a un abordaje integral similar al de la intervención 
que propone nuestro equipo. Se detecta un grupo de cuatro pacientes $(7 \%)$ con enfermedad de presentación severa que son ingresados a protocolo de cirugía para reemplazo articular. En la mayoría de los casos se detecta registro de complemento al abordaje el uso de tratamiento desinflamatorio y analgésico oral y el restante $11.6 \%$ del total de pacientes que se sigue en consulta externa, sólo con tratamiento farmacológico.

La demanda de atención primaria para los adultos mayores de 45 años, el dolor articular y específicamente la osteoartritis es una causa común. Con frecuencia el paciente es referido a la consulta especializada sin una valoración

\begin{tabular}{|c|c|c|}
\hline \multicolumn{3}{|c|}{$\begin{array}{l}\text { Tabla 1: Características generales de las } \\
\text { poblaciones de estudio. } N=120\end{array}$} \\
\hline & $\begin{array}{l}\text { Grupo control } \\
\text { (grupo de } \\
\text { observación), } \\
n=60\end{array}$ & $\begin{array}{c}\text { Grupo de } \\
\text { intervención, } \\
\mathrm{n}=60\end{array}$ \\
\hline & n $(\%)$ & n $(\%)$ \\
\hline \multicolumn{3}{|l|}{ Sexo } \\
\hline Femenino & $48(80.00)$ & $43(71.67)$ \\
\hline Masculino & $12(20.00)$ & $17(28.33)$ \\
\hline Edad (años) & $60.0 \pm 11.8$ & $60.0 \pm 11.8$ \\
\hline \multicolumn{3}{|l|}{ Diagnóstico (grados) } \\
\hline I & $2(3.3)$ & $3(5.0)$ \\
\hline II & $39(65.0)$ & $39(65.0)$ \\
\hline III & $19(31.6)$ & $18(30.0)$ \\
\hline \multicolumn{3}{|l|}{ Tratamiento } \\
\hline Viscosuplementación & $34(58.8)$ & \\
\hline $\begin{array}{l}\text { Cirugía de mínima } \\
\text { invasión }\end{array}$ & $9(15.0)$ & \\
\hline $\begin{array}{l}\text { Tratamiento } \\
\text { farmacológico }\end{array}$ & $7(11.9)$ & \\
\hline $\begin{array}{l}\text { Manejo } \\
\text { multidisciplinario }\end{array}$ & $4(6.8)$ & \\
\hline Reemplazo articular & $6(7.0)$ & \\
\hline
\end{tabular}

consistente en el sector primario. Las directrices en el manejo actual de la osteoartritis recomiendan estrategias básicas de provisión de información, actividad física y ejercicio así como el control de peso, complementadas con el uso racionado de fármacos dentro de un margen de seguridad. De lo anterior recabamos la evidencia de que los pacientes diagnosticados con OA no reciben atención con adecuada directriz basada en las recomendaciones antes mencionadas y que los conducen a depender de otros tratamientos ya sea farmacológicos, invasivos con sustancias externas para la articulación e incluso procedimientos quirúrgicos de manera temprana. La observación en este tipo de atención se muestra en la tabla de características generales.

Diversos estudios señalan que la atención primaria es la línea de abordaje inicial y debe contener intervenciones que involucran al paciente, pero en relación con su entorno para aplicar estrategias en conjunto que puedan reducir el dolor y la discapacidad de la OA. Las personas con enfermedades crónicas necesitan ayuda y apoyo para estimular el autocuidado. Además, los cambios de los hábitos benefician en el corto plazo así como el aumento de la actividad física; sin embargo, es difícil mantener estas acciones en el largo plazo sin la debida asistencia.

La mayoría de las intervenciones muestran la importancia de la pérdida de peso en la mejoría de los síntomas en personas con osteoartritis, principalmente de rodilla, algunos estudios combinan sesiones de ejercicio, observando mejoras en la densidad mineral ósea así como cambios en la estructura de la rodilla. Los diferentes estudios que manejan alguna terapia física parten de la evaluación de las mejoras en los pacientes en un corto plazo (un mes, hasta un año). El conjunto de estos estudios muestra eficacia en las mejoras de los pacientes.

Aunado a esto, el personal médico institucionalizado ante las grandes cargas de trabajo muestra una actitud pesimista con respecto a la enfermedad dando pocas expectativas para el futuro de los enfermos, lo que afecta el

Tabla 2: Características de la población de estudio en el transcurso de la intervención.

\begin{tabular}{|c|c|c|c|c|}
\hline & Cita 1 & Cita 2 & Cita 3 & Cita 4 \\
\hline & $\mathrm{n}=60$ & $\mathrm{n}=60$ & $\mathrm{n}=60$ & $\mathrm{n}=47$ \\
\hline \multicolumn{5}{|l|}{ Antropometría } \\
\hline Peso (kg) & $74.24 \pm 15.65$ & $74.07 \pm 15.53$ & $73.70 \pm 15.56$ & $71.66 \pm 13.46$ \\
\hline Índice de masa corporal & $30.17 \pm 6.15$ & $30.12 \pm 6.18$ & $29.97 \pm 6.20$ & $29.39 \pm 5.43$ \\
\hline Circunferencia de cintura $(\mathrm{cm})$ & $101.46 \pm 13.88$ & $101.41 \pm 13.75$ & $101.19 \pm 13.81$ & $97.70 \pm 15.67$ \\
\hline Circunferencia de cadera $(\mathrm{cm})$ & $106.95 \pm 12.69$ & $107.31 \pm 12.70$ & $107.02 \pm 12.58$ & $105.45 \pm 10.49$ \\
\hline Circunferencia braquial $(\mathrm{cm})$ & $32.94 \pm 4.13$ & $32.89 \pm 4.02$ & $32.78 \pm 4.10$ & $32.12 \pm 3.85$ \\
\hline Circunferencia de pantorrilla $(\mathrm{cm})$ & $37.94 \pm 4.01$ & $38.07 \pm 3.79$ & $37.77 \pm 3.81$ & $37.28 \pm 3.60$ \\
\hline Grasa $(\%)$ & $35.83 \pm 7.78$ & $35.96 \pm 7.40$ & $36.20 \pm 7.98$ & $37.03 \pm 7.45$ \\
\hline \multicolumn{5}{|l|}{ Valoración del dolor } \\
\hline Escala visual análoga (EVA) & $7.18 \pm 2.03$ & $6.05 \pm 1.89$ & $4.97 \pm 2.12$ & $4.46 \pm 2.78$ \\
\hline Flexión & $97.43 \pm 11.62$ & $101.75 \pm 10.18$ & $101.60 \pm 19.41$ & $107.68 \pm 9.50$ \\
\hline Extensión & $7.42 \pm 3.30$ & $6.49 \pm 3.15$ & $5.30 \pm 2.65$ & $4.49 \pm 2.49$ \\
\hline Danniels (fuerza) & $3.50 \pm 0.72$ & $3.69 \pm 0.56$ & $3.86 \pm 0.52$ & $4.05 \pm 0.55$ \\
\hline
\end{tabular}


Tabla 3: Cambio en las medias en las diferentes citas en el transcurso de la intervención.

\begin{tabular}{|c|c|c|c|c|c|c|c|c|c|}
\hline & \multicolumn{2}{|c|}{$\begin{array}{c}\text { Cita } 1 \text { a cita } 2 \\
n=60\end{array}$} & \multirow[b]{2}{*}{$\S \mathrm{p}$} & \multicolumn{3}{|c|}{$\begin{array}{c}\text { Cita } 1 \text { a cita } 3 \\
n=60\end{array}$} & \multicolumn{3}{|c|}{$\begin{array}{c}\text { Cita } 1 \text { a cita } 4 \\
n=47\end{array}$} \\
\hline & $*$ Dif. & $95 \%$ IC & & *Dif. & $95 \%$ IC & $\S \mathrm{p}$ & $*$ Dif. & $95 \%$ IC & $\S p$ \\
\hline \multicolumn{10}{|l|}{ Antropometría } \\
\hline Peso $(\mathrm{kg})$ & -0.175 & $0.647 ;-0.297$ & 0.461 & -0.543 & $-1.103 ; 0.017$ & 0.057 & -0.649 & $-1.388 ; 0.090$ & 0.084 \\
\hline Índice de masa corporal & -0.054 & $-0.236 ; 0.128$ & 0.554 & -0.203 & $-0.415 ; 0.010$ & 0.061 & -0.234 & $-0.511 ; 0.442$ & 0.097 \\
\hline Circunferencia de cintura $(\mathrm{cm})$ & -0.047 & $-0.796 ; 0.703$ & 0.901 & -0.267 & $-0.913 ; 0.379$ & 0.412 & -2.287 & $-5.686 ; 1.112$ & 0.182 \\
\hline Circunferencia de cadera $(\mathrm{cm})$ & 0.362 & $-0.107 ; 0.831$ & 0.128 & 0.065 & $-0.475 ; 0.605$ & 0.811 & -0.264 & $-1.084 ; 0.556$ & 0.520 \\
\hline Circunferencia braquial $(\mathrm{cm})$ & -0.045 & $-0.222 ; 0.132$ & 0.614 & -0.153 & $-0.342 ; 0.035$ & 0.109 & -0.332 & $-0.641 ;-0.023$ & 0.036 \\
\hline Circunferencia de pantorrilla $(\mathrm{cm})$ & 0.122 & $-0.089 ; 0.332$ & 0.252 & -0.173 & $-0.402 ; 0.055$ & 0.135 & -0.268 & $-0.583 ; 0.047$ & 0.093 \\
\hline Grasa $(\%)$ & 0.129 & $-1.077 ; 1.335$ & 0.831 & 0.377 & $-0.366 ; 1.120$ & 0.315 & 0.968 & -0.0752 .011 & 0.068 \\
\hline \multicolumn{10}{|l|}{ Valoración del dolor } \\
\hline Escala visual análoga & -1.133 & $-1.369 ;-0.898$ & $<0.000$ & -2.217 & $-2.612 ;-1.821$ & $<0.000$ & -2.743 & $-3.535 ;-1.951$ & $<0.000$ \\
\hline Flexión & 4.441 & $2.813 ; 6.068$ & $<0.000$ & 4.259 & $-1.125 ; 9.642$ & 0.119 & 8.950 & $6.015 ; 11.885$ & $<0.000$ \\
\hline Extensión & -0.932 & $-1.500 ;-0.364$ & 0.002 & -2.211 & $-2.903 ;-1.518$ & $<0.000$ & -2.216 & $-3.288 ;-1.144$ & 0.000 \\
\hline Danniels (fuerza) & 0.186 & $0.541 ; 0.319$ & 0.007 & 0.386 & $0.222 ; 0.550$ & $<0.000$ & 0.525 & $0.285 ; 0.765$ & 0.000 \\
\hline
\end{tabular}

Tabla 4: Efectos de la intervención; modelos para las variables de valoración del dolor.

\begin{tabular}{|c|c|c|c|c|}
\hline Citas & 1 & 2 & 3 & 4 \\
\hline \multicolumn{5}{|l|}{ EVA } \\
\hline $\begin{array}{l}\text { MD* } \\
\beta \\
\text { IC } 95 \% \\
p\end{array}$ & 7.189 & $\begin{array}{c}6.056 \\
-1.133333 \\
-1.494252 ;-0.772 \\
<0.000\end{array}$ & $\begin{array}{c}4.972 \\
-2.216667 \\
-2.577586 ;-1.856 \\
<0.000\end{array}$ & $\begin{array}{c}4.403 \\
-2.786244 \\
-3.218132 ;-2.354 \\
<0.000\end{array}$ \\
\hline \multicolumn{5}{|l|}{ Flexión } \\
\hline $\begin{array}{l}\text { MD* } \\
\beta \\
\text { IC } 95 \% \\
p\end{array}$ & 97.561 & $\begin{array}{c}101.943 \\
4.383 \\
0.443 ; 8.322 \\
0.029\end{array}$ & $\begin{array}{c}101.829 \\
4.268 \\
0.310 ; 8.225 \\
0.035\end{array}$ & $\begin{array}{l}106.866 \\
9.305 \\
4.914 ; 13.695 \\
<0.000\end{array}$ \\
\hline \multicolumn{5}{|c|}{ Extensión } \\
\hline $\begin{array}{l}\text { MD* } \\
\beta \\
\text { IC } 95 \% \\
p\end{array}$ & 7.357 & $\begin{array}{c}6.425 \\
-0.932 \\
-1.587 ;-0.277 \\
0.005\end{array}$ & $\begin{array}{c}5.165 \\
-2.192 \\
-2.854 ;-1.531 \\
<0.000\end{array}$ & $\begin{array}{c}4.961 \\
-2.396 \\
-3.151 ;-1.151 \\
<0.000\end{array}$ \\
\hline \multicolumn{5}{|c|}{ Danniels } \\
\hline $\begin{array}{l}\text { MD* } \\
\beta \\
\text { IC } 95 \% \\
p\end{array}$ & 3.501 & $\begin{aligned} & 3.691 \\
& 0.191 \\
& 0.540 ; 0.327 \\
&<0.000\end{aligned}$ & $\begin{aligned} & 3.879 \\
& 0.378 \\
0.240 ; 0.516 & \\
< & 0.000\end{aligned}$ & $\begin{array}{c}4.038 \\
0.538 \\
0.384 ; 0.691 \\
<0.000\end{array}$ \\
\hline
\end{tabular}

ánimo y la esperanza de una mejora en la calidad de vida. Durante el desarrollo de este proyecto nos encontramos con verdaderas dificultades para introducir el programa en las diferentes clínicas que fueron elegidas para dicho propósito, el personal de salud se muestra reacio a la colaboración con grupos organizados de salud que buscan cambiar el abordaje tradicional del tratamiento de esta enfermedad.

Un modelo de atención integral desde la atención primaria, que incluye recomendaciones en materia de nutrición y salud física con rehabilitación a partir de programas para llevar a cabo en casa, ofrece un trata- 
miento que se extiende hasta los ámbitos familiares y personales de cada individuo y esto se puede alcanzar desde las fases tempranas de la enfermedad y el médico de primer contacto.

Si bien en este primer análisis de resultados el control de peso no fue significativo estadísticamente, tiene una tendencia a ir disminuyendo en el transcurso de la intervención, lo que favorece la mejoría de los síntomas, encontrándose relacionadas estas variables. Por lo anterior, creemos que el abordaje multidisciplinario sí es fundamental en la percepción que el paciente tiene de sí mismo al recibir estímulo y apoyo en las tareas encomendadas para lograr el cambio de hábitos.

\section{Conclusiones}

La atención primaria o de primer contacto puede tener un impacto en la distribución de los recursos humanos, económicos y materiales. Los pacientes atendidos por medio de este tipo de intervención logran aprender y toman con seriedad su papel predominante en el autocuidado de la salud al sentirse apoyados con el máximo de explotación de su entorno.

Los médicos de atención primaria tienen mayor conocimiento de la enfermedad para mejorar las acciones preventivas. Los pacientes tuvieron precepción de mejoría después de recibir la orientación y adherirse a los programas recomendados en materia de rehabilitación, nutrición, sicología y consejo social.

En la clínica de primer nivel de atención se reconoce la necesidad de desarrollar programas encaminados a la educación de los pacientes en materia de salud articular y lo obligatorio que resulta el cambio de hábitos en una cultura de autocuidado. Con relación a la atención médica, los programas deben incluir la sensibilización del personal de salud para retomar la importancia que tiene la salud articular y su relación con el impacto biopsicosocial que éste tiene en los pacientes cuando inician los síntomas de la enfermedad.

Es imperativo cambiar el enfoque de atención que reciben los pacientes con OA al interior del ISSSTE. Esperamos que los resultados de un manejo integral desde la atención primaria fortalezcan la calidad de atención y la mejor distribución de los recursos. Pretendemos con estas acciones mejorar la calidad de vida de los pacientes con educación, atención, intervención y seguimiento en el cambio de un estilo de vida saludable.

\section{Referencias}

1. Kloek CJJ, Dongen JM, de Bakker DH, Bossen D, Dekker J, Veenhof C. Cost-effectiveness of a blended physiotherapy in patients with hip and/or knee osteoarthritis: a cluster randomized controlled trial. BMC Public Health. 2018; 18(1): 1082. doi: 10.1186/s12889-018-5975-7.

2. Mora JC, Przkora R, Cruz-Almeida Y. Knee osteoarthritis: pathophysiology and current treatment modalities. J Pain Res. 2018; 11: 2189-96. doi: 10.2147/JPR.S154002.

3. Kuntz AB, Chopp-Hurley JN, Brenneman EC, Karampatos S, Wiebenga EG, Adachi JD, et al. Efficacy of a biomechanically-based yoga exercise program in knee osteoarthritis: a randomized controlled trial. PLoS One. 2018; 13(4): e0195653. doi: 10.1371/journal. pone.0195653.

4. Kabiri S, Halabchi F, Angoorani H, Yekaninejad S. Comparison of three modes of aerobic exercise combined with resistance training on the pain and function of patients with knee osteoarthritis: A randomized controlled trial. Phys Ther Sport. 2018; 32: 22-8. doi: 10.1016/j.ptsp.2018.04.001.

5. Sit RWS, Chan KKW, Yip BHK, Zhang DD, Reeves KD, Chan YH, et al. Clinical effectiveness of patella mobilisation therapy versus a waiting list control for knee osteoarthritis: a protocol for a pragmatic randomised clinical trial. BMJ Open. 2018; 8(3): e019103. doi: 10.1136/bmjopen-2017-019103.

6. Yan A, Zhang K, Qin WK, Guo JZ, Li LH, Chen M et al. Clinical effects of rehabilitation exercise in the treatment of knee osteoarthritis based on the theory of "treating muscle to the bone". Zhongguo Gu Shang. 2017; 30(8): 731-4. doi: 10.3969/j.issn.1003-0034.Chino.

7. Isaramalai SA, Hounsri K, Kongkamol C, Wattanapisitkul P, Tangadulrat N, Kaewmanee $\mathrm{T}$, et al. Integrating participatory ergonomic management in non-weight-bearing exercise and progressive resistance exercise on self-care and functional ability in aged farmers with knee osteoarthritis: a clustered randomized controlled trial. Clin Interv Aging. 2018; 13: 101-8. doi: 10.1186/ s12891-018-1968-Z.

8. Lai Z, Zhang Y, Lee S, Wang L. Effects of strength exercise on the knee and proprioception of the ankle of individuals with knee osteoarthritis. Res Sports Med. 2018; 26(2): 138-46. doi: 10.1080/15438627.2018.1431541.

9. Chang FH, Jette AM, Slavin MD, Baker K, Ni P, Keysor JJ. Detecting functional change in response to exercise in knee osteoarthritis: a comparison of two computerized adaptive tests. BMC Musculoskelet Disord. 2018; 19(1): 29. doi: 10.1186 / s12891-018-1942-9.

10. Jackson H, Barnett LA, Jordan KP, Dziedzic KS, Cottrell E, Finney $\mathrm{AG}$, et al. Patterns of routine primary care for osteoarthritis in the UK: a crosssectional electronic health records study. BMJ Open. 2017; 7(12): e019694. doi: 10.1136/bmjopen-2017-019694.

11. Robbins SR, Melo LRS, Urban H, Deveza LA, Asher R, Johnson VL, Hunter DJ. Stepped care approach for medial tibiofemoral osteoarthritis (STrEAMline): protocol for a randomised controlled trial. BMJ Open. 2017; 7(12): e018495. doi: 10.1136/bmjopen-2017-018495.

12. Gomiero AB, Kayo A, Abraao M, Peccin MS, Grande AJ, Trevisani VF. Sensory-motor training versus resistance training among patients with knee osteoarthritis: randomized single-blind controlled trial. Sao Paulo Med J. 2018; 136(1): 44-50. doi: 10.1590/15163180.2017.0174100917.

13. Hall M, Hinman RS, Van der Esch M, Van der Leeden M, Kasza J, et al. Is the relationship between increased knee muscle strength and improved physical function following exercise dependent on baseline physical function status? Arthritis Res Ther. 2017; 19(1): 271. doi: 10.1186/s13075-017-1477-8.

14. Brand A, Klopfer-Kramer I, Morgenstern M, Kroger I, Michel B, Thannheimer A, et al. Effects of knee orthosis adjustment on biomechanical performance and clinical outcome in patients with medial knee osteoarthritis. Prosthet Orthot Int. 2017; 41(6): 587-94. doi: 10.1177/0309364617691623.

15. Mazloum V, Rabiei P, Rahnama N, Sabzehparvar E. The comparison of the effectiveness of conventional therapeutic exercises and Pilates on pain and function in patients with knee osteoarthritis. Complement Ther Clin Pract. 2018; 31: 343-8. doi: 10.1016/j.ctcp.2017.10.008.

16. Hausmann LRM, Youk A, Kwoh CK, Ibrahim SA, Hannon MJ, Weiner DK, et al. Testing a positive psychological intervention for osteoarthritis. Pain Med. 2017; 18(10): 1908-20. doi: 10.1093/pm/pnx141.

17. Lopez HL, Habowski SM, Sandrock JE, Raub B, Kedia A, Bruno EJ, Ziegenfuss TN. Effects of dietary supplementation with a standardized aqueous extract of Terminalia chebula fruit (AyuFlex(®)) on joint mobility, comfort, and functional capacity in healthy overweight subjects: a randomized placebo-controlled clinical trial. BMC Complement Altern Med. 2017; 17(1): 475. doi: 10.1186/s12906-017-1977-8.

18. Fernandes GS, Sarmanova A, Warner S, Harvey H, Akin-Akinyosoye $\mathrm{K}$, Richardson $\mathrm{H}$, et al. Knee pain and related health in the community 
study (KPIC): a cohort study protocol. BMC Musculoskelet Disord. 2017; 18(1): 404. doi: 10.1186/s12891-017-1761-4.

19. Lai Z, Wang X, Lee S, Hou X, Wang L. Effects of whole body vibration exercise on neuromuscular function for individuals with knee osteoarthritis: study protocol for a randomized controlled trial. Trials. 2017; 18(1): 437. doi: 10.1186/s13063-017-2170-6.

20. Schell J, Scofield RH, Barrett JR, Kurien BT, Betts N, Lyons TJ, Zhao YD, Basu A. Strawberries improve pain and inflammation in obese adults with radiographic evidence of knee osteoarthritis. Nutrients. 2017; 9(9): 949. doi: 10.3390/nu9090949.

21. Swaminathan V, Parkes MJ, Callaghan MJ, O’Neill TW, Hodgson R, Gait $\mathrm{AD}$, et al. With a biomechanical treatment in knee osteoarthritis, less knee pain did not correlate with synovitis reduction. BMC Musculoskelet Disord. 2017; 18(1): 347. doi: 10.1186/s12891-0171691-1.

22. Bartels EM, Henrotin Y, Bliddal H, Centonze P, Henriksen M. Relationship between weight loss in obese knee osteoarthritis patients and serum biomarkers of cartilage breakdown: secondary analyses of a randomised trial. Osteoarthritis Cartilage. 2017; 25(10): 1641-6. doi: 10.1016/j.joca.2017.06.009.

23. Bossen D, Veenhof C, Van Beek KE, Spreeuwenberg PM, Dekker J, De Bakker DH. Effectiveness of a web-based physical activity intervention in patients with knee and/or hip osteoarthritis: randomized controlled trial. J Med Internet Res. 2013; 15(11): e257. doi: 10.2196/jmir.2662.

24. Krauss I, Mueller G, Haupt G, Steinhilber B, Janssen P, Jentner N, Martus P. Effectiveness and efficiency of an 11-week exercise intervention for patients with hip or knee osteoarthritis: a protocol for a controlled study in the context of health services research. BMC Public Health. 2016; 16: 367. doi: 10.1186/s12889-016-3030-0.

25. Bartels EM, Christensen R, Christensen P, Henriksen M, Bennett A, Gudbergsen H, et al. Effect of a 16 weeks weight loss program on osteoarthritis biomarkers in obese patients with knee osteoarthritis: a prospective cohort study. Osteoarthritis Cartilage. 2014; 22(11): 1817-25. doi: 10.1016/j.joca.2014.07.027. 